\title{
Box- and peanut-shaped bulges
}

\section{A new class of bulges: Thick Boxy Bulges}

\author{
R. Lütticke ${ }^{1,2}$, M. Pohlen ${ }^{1,3}$, and R.-J. Dettmar ${ }^{1}$ \\ 1 Astronomical Institute, Ruhr-Universität Bochum, 44780 Bochum, Germany \\ 2 Department of Computer Science, Intelligent Information and Communication Systems, FernUniversität Hagen, \\ Universitätsstr. 1, 58084 Hagen, Germany \\ 3 Instituto de Astrofisica de Canarias, La Laguna, 38200 Tenerife, Spain
}

Received 3 December 2001 / Accepted 19 December 2003

\begin{abstract}
Inspecting all 1224 edge-on disk galaxies larger than 2' in the RC3 on Digitized Sky Survey (DSS) images (Lütticke et al. 2000a) we have found several galaxies with extraordinary bulges meeting two criteria: they are box shaped and large in respect to the diameters of their galaxies. These bulges are often disturbed, show frequently prominent irregularities and asymmetries, and some possess possible merger remnants or merging satellites. For these bulges we have introduced the term "Thick Boxy Bulges" (TBBs). About 2\% of all disk galaxies (S0-Sd), respectively $4 \%$ of all galaxies with box- and peanut-shaped (b/p) bulges, belong to this class of galaxies. Using multicolour CCD and NIR data we have enlarged and followed up our sample of nearly 20 galaxies with a TBB. The disturbed morphology of a large fraction of these galaxies shows that many of the TBB galaxies are not dynamically settled. For the TBBs the extent of the box shape seems to be too large to result from a normal bar potential. Therefore we conclude that two classes of b/p bulges exist with different origins. While most ( 96\%) b/p bulges can be explained by bars alone (Lütticke et al. 2000b), the extended boxy structures of TBBs result most likely from accreted material by infalling satellite companions (soft merging).
\end{abstract}

Key words. galaxies: bulges - galaxies: evolution - galaxies: interactions - galaxies: spiral - galaxies: statistics galaxies: structure

\section{Introduction}

Recent statistics of $b / p$ bulges have revealed that nearly half of all disk galaxies (S0-Sd) have bulges which differ from an elliptical shape (Lütticke et al. 2000a, hereafter Paper I). Several studies have shown that there is strong evidence that bar instabilities and resonances are at work in most cases producing these b/p bulges (Combes et al. 1990; Raha et al. 1991; Kuijken \& Merrifield 1995; Bureau \& Freeman 1999; Lütticke et al. 2000b, hereafter Paper II; Athanassoula \& Misiriotis 2002; Patsis et al. 2002b). In addition to spontaneous bar instabilities in disks, such instabilities can also be triggered by interactions followed by accretions of material (Noguchi 1987; Gerin et al. 1990; Walker et al. 1996). The evolutionary scenario - interaction, possible accretion, and then the formation of a bar as progenitor of $b / p$ structures - is supported by observations and simulations (Fisher et al. 1994; Mihos et al. 1995). Observational evidence for accreted material in $b / p$ bulges is also found by Bureau \& Freeman (1999) for a few b/p bulges in a kinematical study.

On the other side several theories exist for the origin of $\mathrm{b} / \mathrm{p}$ bulges, explaining their non elliptical shape directly by

Send offprint requests to: $\mathrm{R}$. Lütticke,

e-mail: rainer. luetticke@fernuni-hagen.de merger events. Binney \& Petrou (1985) and Rowley (1988) proposed mergers of two disk galaxies as a formation process of $b / p$ bulges. However, the precise alignment of the spin and orbital angular momenta of the two galaxies require very special conditions. Therefore such mergers would be quite rare (Bureau 1998). The accretion of satellite galaxies could be a more common process to form $b / p$ bulges (Binney \& Petrou 1985; Whitmore \& Bell 1988). For such a scenario the satellite must have an oblique impact angle (Whitmore \& Bell 1988) and a mass, which is large enough to produce morphological changes and small enough not to disrupt the stellar disk (Barnes 1992; Hernquist 1993). The theories for a possible origin of $\mathrm{b} / \mathrm{p}$ bulges based on accretion events are supported by $N$-body simulations of Hernquist \& Quinn (1989). Additionally, Dettmar (1989) and Dettmar \& Barteldrees (1990a) pointed out that some boxy bulges with large radial extent cannot be explained within an evolutionary scenario of $b / p$ bulges based on bars and that asymmetries and irregularities in a few galaxies with boxy bulges rather hint to a recent merger event.

A third theory for the formation of $b / p$ bulges has not found further support in the literature, because the proposed external cylindrically symmetric torques (May et al. 1985) as origin of 
such bulges are difficult to relate to some astrophysical counterparts (Combes et al. 1990).

Whereas our previous study (Paper II) revealed that the aspect angle of bars is the main cause for the shape of bulges, we present here a sample of extraordinary b/p bulges to investigate for the first time on a large basis of observational data the relevance of theories of merger/accretion scenarios inducing a special class of $b / p$ bulges. In this way constraints on theories about secular evolution of bulges (e.g. Combes 2000; Pfenniger 1999) can be found, since it is still open what roles play bar formation/destruction and accretion events/soft mergers for secular dynamical evolution. Detailed studies of the structure of $\mathrm{b} / \mathrm{p}$ bulges are needed to discriminate between the importance of the proposed building processes of $\mathrm{b} / \mathrm{p}$ bulges.

\section{Data}

\subsection{Full data base}

We have compiled a list of $b / p$ bulges in edge-on galaxies consisting of two parts:

1. RC3-survey: all galaxies out of the RC3 (Third Reference Catalogue of Bright Galaxies, de Vaucouleurs et al. 1991) with $D_{25}>2^{\prime}$ and $\log R_{25} \geq 0.35,0.30$ for $\mathrm{S} 0$ galaxies respectively.

The RC3-survey is complete over the whole sky and consists of 1224 galaxies ranging from S0 to $\mathrm{Sd}(-3.5<$ $T<7.5)$. These galaxies are visually inspected using the DSS $1^{1} .734$ of them have bulges for which the shape can be classified (Paper I). Additionally, for 76 galaxies of the RC3-survey we obtained optical and for 56 galaxies NIR follow-up images.

2. Serendipitous survey: 51 galaxies outside the selection criteria of the RC3-survey with imaging data taken from several different projects.

In detail this are: two edge-on galaxies (optical images) with an axis ratio outside the selection limits, 48 galaxies (44 optical and four NIR images) with a diameter smaller than $2^{\prime}$, and one galaxy smaller than $2^{\prime}$ and an axis ratio outside the limits (VLT image ${ }^{2}$ ).

The observations of the optical and NIR data were obtained in several runs. Data reduction is presented elsewhere (optical: Barteldrees \& Dettmar 1994; Pohlen et al. 2000, Paper I; Pohlen 2001; NIR: Paper II).

\subsection{Sample selection of the TBBs}

From this survey we have extracted a list of objects that fall into a new category of disk galaxies hosting large and boxy bulges. Their morphological appearance suggests to call them "Thick Boxy Bulges" (TBBs). Following the shape definitions of bulge types (Paper I) TBBs are classified as type 2 (box-shaped bulge) or 3 (bulge is close to box-shaped, not elliptical). Since

\footnotetext{
${ }^{1}$ http://archive.eso.org/dss/dss

2 http://www.eso.org/outreach/info-events/ut1fl/ astroim-galaxy.html
}

"normal" bulges have a relative size of BUL/ $D_{25}=0.22$ $\left(\mathrm{BUL}=\right.$ bulge length ${ }^{3}$, values derived in Paper II ranging from 0.10 to 0.42 including S0-Scd galaxies), we define a bulge as "thick" if BUL/ $D_{25}>0.5$ or if bulge and disk component cannot be clearly separated due to the large extent of the bulge. Bulges of 13 galaxies out of the RC3-survey and 6 galaxies from the serendipitous survey fall into the class of TBBs (Table 1). Compared to the preliminary versions of this catalogue (Dettmar \& Lütticke 1999; Lütticke \& Dettmar 1999) some minor changes of the list of TBBs are made due to the homogeneous definition of thickness and a reclassification of the bulge types with data of higher quality.

\section{Results}

\subsection{Morphology}

TBBs show frequently extra features (e.g. twists of the isophotes) and large scale asymmetries. They can be divided in asymmetric (58\%) and rather symmetric (42\%) members of the TBB class by their morphological appearance and degree of peculiarities (Table 1). Examples for galaxies excluded from the list of TBBs (see below: non-TBBs) clarify the characteristics of the TBBs. For all DSS candidates reobserved (e.g. Fig. 1) the suspected peculiarities are confirmed. It is noticeable that all galaxies with a TBB (excluding the S0 galaxy ESO 322-100) possess a prominent dust lane which is often warped.

Asymmetric TBBs: the eleven asymmetric members of the class of TBBs possess a variety of individual features. However, the description of their morphology in detail depends on the quality of the data. Therefore it is natural that faint structures in galaxies investigated only with DSS or low quality CCD data remain undetected. The detailed description of all the peculiarities of the individual TBBs presents a full overview about their morphology (Sect. 3.2).

Rather symmetric TBBs: there are eight out of the 19 TBBs catalogued in Table 1, which show only small asymmetries and nearly no peculiarities. (e.g. Figs. 1 and 17). However, they fulfill the two criteria thickness and boxiness for a TBB. In these galaxies there is no boxy envelope visible and the extent of the boxiness is often limited to the inner parts of the bulge. Only the weakly asymmetric tilted disks in UGC 3458 and ESO 506-003 (Fig. 1) and the slightly asymmetry of the merged disk/bulge component of ESO 514-005 are remarkable.

Examples for non-TBBs: there exists three groups of galaxies which could be associated with the class of TBB galaxies, but do not belong to this class, since they do not fulfill the criteria of the TBBs.

The first group includes prominent boxy and a few peanut bulges in edge-on galaxies. They possess partly also irregularities, but their bulges are not large enough to influence the shape

\footnotetext{
3 The bulge length is marked by an increasing light distribution compared to the exponential disk in a radial surface brightness profile (excluding a possible bar).
} 
Table 1. Catalogue of galaxies with a TBB.

\begin{tabular}{|c|c|c|c|c|c|c|c|c|c|c|c|}
\hline $\begin{array}{c}(1) \\
\text { Galaxy }\end{array}$ & $\begin{array}{c}(2) \\
\text { RA } \\
(2000)\end{array}$ & $\begin{array}{c}(3) \\
\text { Dec } \\
(2000)\end{array}$ & \begin{tabular}{l}
\multicolumn{1}{c}{$(4)$} \\
Morph. \\
type
\end{tabular} & $\begin{array}{c}(5) \\
D_{25} \\
{\left[{ }^{\prime \prime}\right]}\end{array}$ & $\begin{array}{c}(6) \\
\text { Survey }\end{array}$ & $\begin{array}{c}\text { (7) } \\
\text { Image } \\
\text { quality }\end{array}$ & $\begin{array}{c}(8) \\
\text { Bulge } \\
\text { type }\end{array}$ & $\begin{array}{c}(9) \\
\text { Asym- } \\
\text { metry }\end{array}$ & $\begin{array}{l}(10) \\
n\end{array}$ & $\begin{array}{c}(11) \\
w\end{array}$ & $\begin{array}{c}(12) \\
\text { Group }\end{array}$ \\
\hline ESO 013-012 & 0107 & -8018 & S0a & 165 & RCS & - & 2 & & & 1 & - \\
\hline NGC 1030 & 0240 & +1802 & SO* & 95 & SDS & fair & 3 & $x$ & $2^{d}$ & 2 & PPS2 173 \\
\hline NGC 1055 & 0242 & +0027 & $\mathrm{Sb}$ & 455 & RCS & fair & 2 & $x$ & $2^{d}$ & 2 & LGG 73 \\
\hline NGC 1589 & 0431 & +0052 & $\mathrm{Sab}$ & 190 & RCS & good & 2 & $x$ & 2 & 2 & LGG 117 \\
\hline UGC 3458 & 0626 & +6444 & $\mathrm{Sb}$ & 144 & RCS & good & 3 & & 3 & 2 & $?$ \\
\hline ESO 494-022 ${ }^{a}$ & 0806 & -2449 & $\mathrm{Sa}$ & 134 & RCS & - & 3 & & & 1 & $?$ \\
\hline NGC 3573 & 1111 & -3652 & SOa & 218 & RCS & good & 3 & $x$ & & 2 & LGG 229 \\
\hline NGC 4224 & 1216 & +0727 & $\mathrm{Sa}$ & 154 & RCS & - & 3 & & & 1 & LGG 281 \\
\hline ESO 506-003 & 1222 & -2505 & $\mathrm{Sab}$ & 85 & SDS & good & 3 & & 2 & 2 & $?$ \\
\hline ESO 322-100 & 1249 & -4127 & So & 56 & SDS & good & 2 & & 2 & 1 & LGG 305 \\
\hline ESO 383-005 & 1329 & -3416 & $\mathrm{Sbc}$ & 213 & RCS & good & 2 & $x$ & 3 & 2 & LGG 353 \\
\hline ESO 510-013 & 1355 & -2647 & $\mathrm{Sa}$ & 117 & SDS & good & 3 & $x$ & & 2 & - \\
\hline NGC 5719 & 1441 & -0019 & $\mathrm{Sab}$ & 194 & RCS & - & 3 & $x$ & & 2 & LGG 386 \\
\hline UGC 9759 & 1511 & +5521 & $\mathrm{~S} 0 \mathrm{a}^{* b}$ & 120 & RCS & fair & 2 & & 2 & 1 & LGG 395 \\
\hline ESO 514-005 & 1519 & -2349 & $\mathrm{Sa}$ & 125 & RCS & good & 2 & & 1 & 1 & $?$ \\
\hline UGC 10205 & 1607 & +3006 & $\mathrm{Sa}$ & 87 & SDS & fair & 3 & $x$ & $4^{c}$ & 3 & $?$ \\
\hline IC 4745 & 1842 & -6456 & Sab & 128 & RCS & good & 2 & $x$ & 2 & 3 & LGG 422 \\
\hline IC 4757 & 1844 & -5710 & SOa & 83 & SDS & good & 2 & $x$ & 3 & 1 & $?$ \\
\hline NGC 7183 & 2202 & -1855 & So & 228 & RCS & - & 3 & $x$ & $1^{d}$ & 2 & - \\
\hline
\end{tabular}

Notes to the table:

Columns (2) and (3): RA and Dec are rounded.

Column (4): morphological types marked with an asterisk * from Skiff (1999), others from RC3.

Column (5): $D_{25}$ in arcsec from RC3.

Column (6): RCS: detected in the RC3-survey. SDS: detected in the serendipitous survey (Sect. 2.1).

Column (7): verified by optical CCD or NIR follow-up with "good" or "fair" quality of the data.

Column (8): bulge type as defined in Paper I.

Column (9): asymmetric TBBs are marked $(\times)$.

Column (10): shape parameter of the luminosity profile measured along the minor axis (Sect. 3.3).

Column (11): interaction index as defined in van den Bergh et al. (1996) (Sect. 3.4).

Column (12): membership in a group from NED: LGG numbers from Garcia (General study of group membership 1993), ?=galaxies are fainter than the limit of completeness $\left(B_{0}<14 \mathrm{mag}\right)$ in the sample of Garcia (1993) (Sect. 3.4).

${ }^{a}$ : galaxy is affected by foreground stars due to position behind the Milky Way.

$b$ : S0 possible.

${ }^{c}:$ two components can be detected.

${ }^{d}$ : values derived by Vergani et al. (2001b).

of the whole galaxy so that disk and bulge are separated and $\mathrm{BUL} / D_{25}$ is smaller, although sometimes not much, than 0.5. Such cases are e.g. NGC 482 and IC 4767. However, since decomposition of disk and bulge for S0 galaxies is not always clear cut, BUL cannot always definitely determined.

Secondly, there are thick bulges having nearly the same extent along the major axis as the disks of these galaxies (also in galaxies as late as $\mathrm{Sb}$ ) and BUL/ $D_{25}$ is larger than 0.5 , but these bulges are more elliptical (bulge type 4) than boxy. Therefore we call them TSBs (Thick Spheroidal Bulges). In our survey we have found five TSBs: NGC 4594 (Sombrero Galaxy), NGC 6948 (Fig. 2), NGC 7123, NGC 7814, and ESO 142-19. All these galaxies are rather symmetric. Regarding the 734 galaxies with a classifiable bulge out of our RC3-survey (Sect. 2.1) we get the result that $1.8 \%$ of the galaxies host a TBB and only $0.7 \%$ a TSB.
Nearly disrupted galaxies, although having large scale boxy structures, are also excluded from the class of galaxies with a TBB, e.g. NGC 660 (Fig. 3) and NGC 2992. These galaxies are strongly disturbed and can hardly be defined as disk galaxies anymore. In fact they are in-between disks and completely irregular galaxies.

Dividing the 716 galaxies with a normal sized (non-thick) bulge out of the RC3-survey in asymmetric and symmetric in the same way as done for the TBBs we find large differences between the samples. The fraction of asymmetric TBBs is $54 \%$ whereas the fraction of asymmetric TSBs is zero and of asymmetric normal sized bulges $2.7 \%$ (74\% of them are boxy and $26 \%$ non-boxy) (Table 2 ). The latter values seem small compared to other studies but we use a very strict definition of asymmetry and have neglected the galaxies with unclassifiable bulges in the RC3-survey due to very strong perturbations/disruption. 

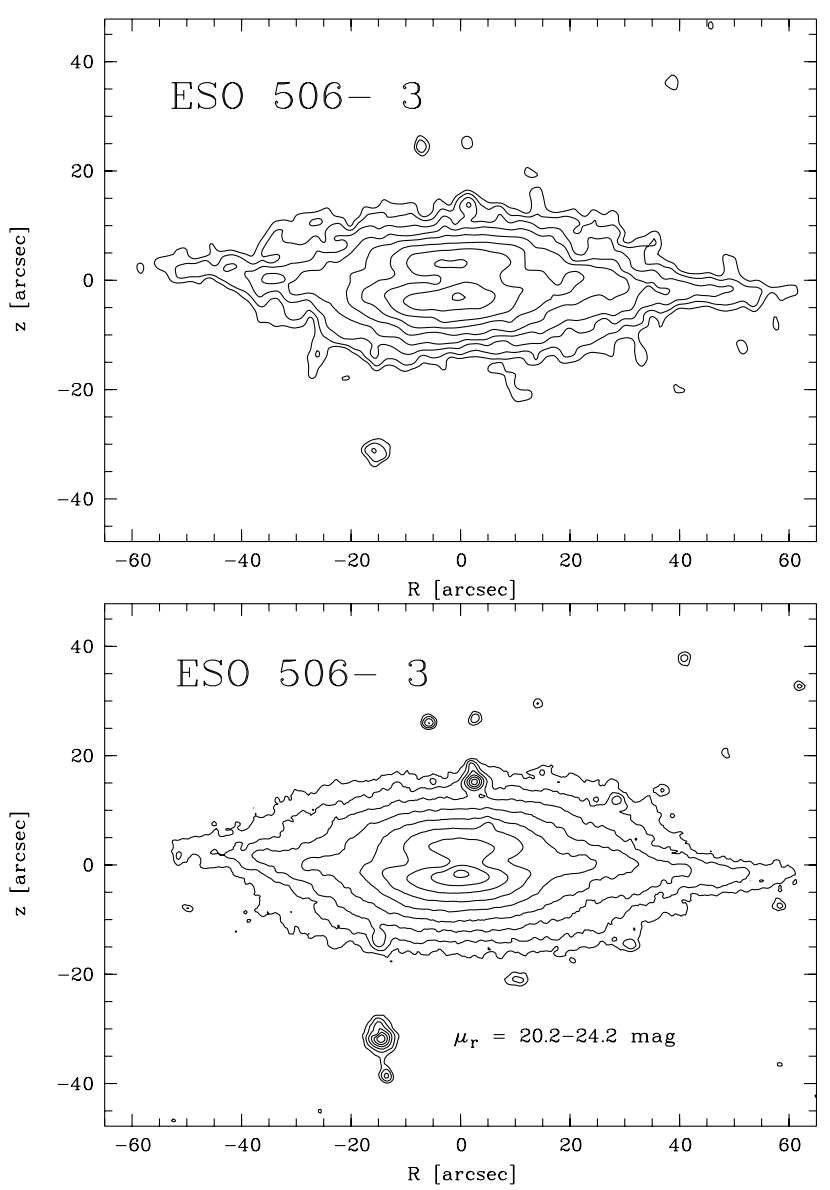

Fig. 1. ESO 506-003. Top: contour plot from DSS. Bottom: for comparison CCD follow-up. ESO/2.2 m, $30 \mathrm{~min}$ in $r$.

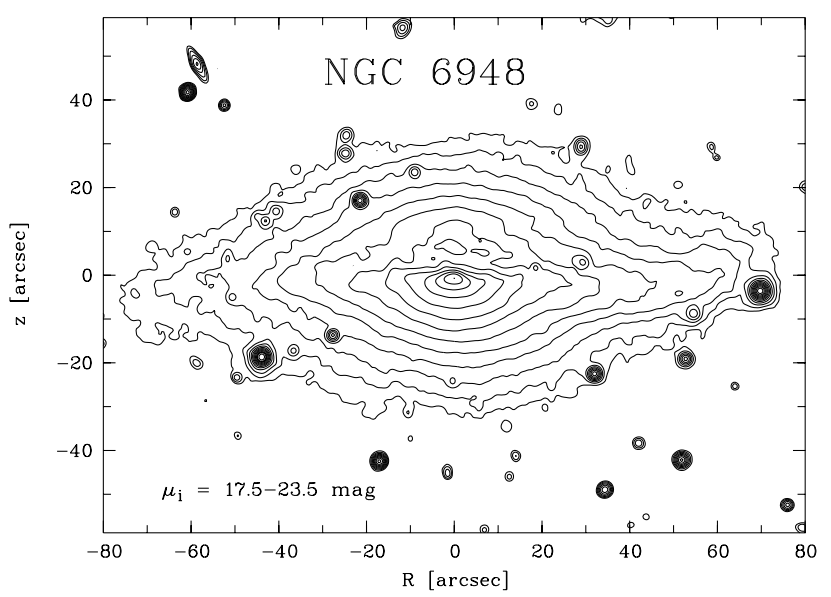

Fig. 2. A galaxy with a TSB: NGC 6948 has a prominent large bulge (BUL/ $D_{25} \sim 0.6$ ), but with elliptical shape. The disk is warped, but in comparison to the asymmetric TBBs the asymmetry is rather weak. $\mathrm{ESO} / 2.2 \mathrm{~m}, 30 \mathrm{~min}$ in $i$.

\subsection{Individual notes on the asymmetric TBBs}

In the following section we describe in detail the eleven prominent TBBs:

NGC 1030 (Fig. 4) has a rectangular shape with merged bulge and disk component and the asymmetries grow with distance from the major and minor axis.

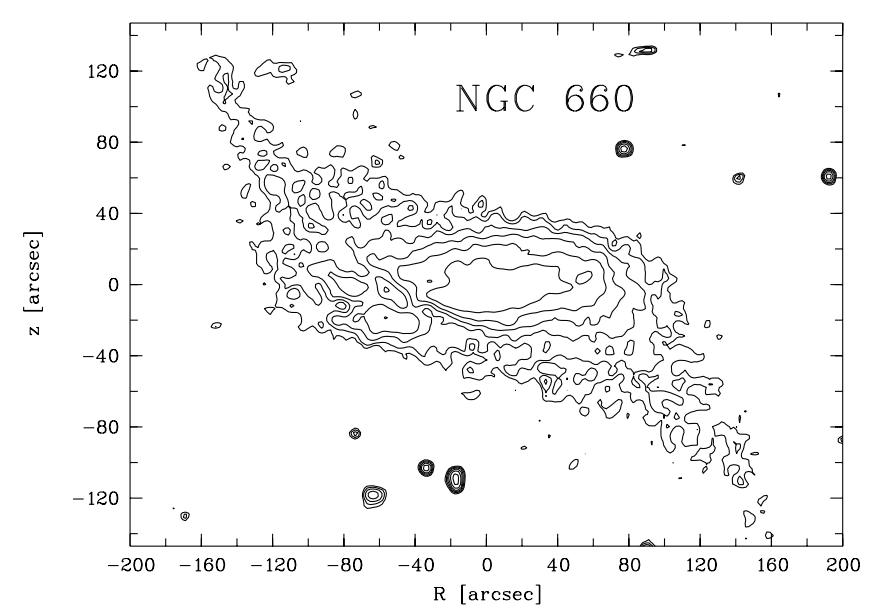

Fig. 3. Excluded galaxy (non-TBB): NGC 660 possesses boxy structures, but it is nearly disrupted. Contour plot from DSS.

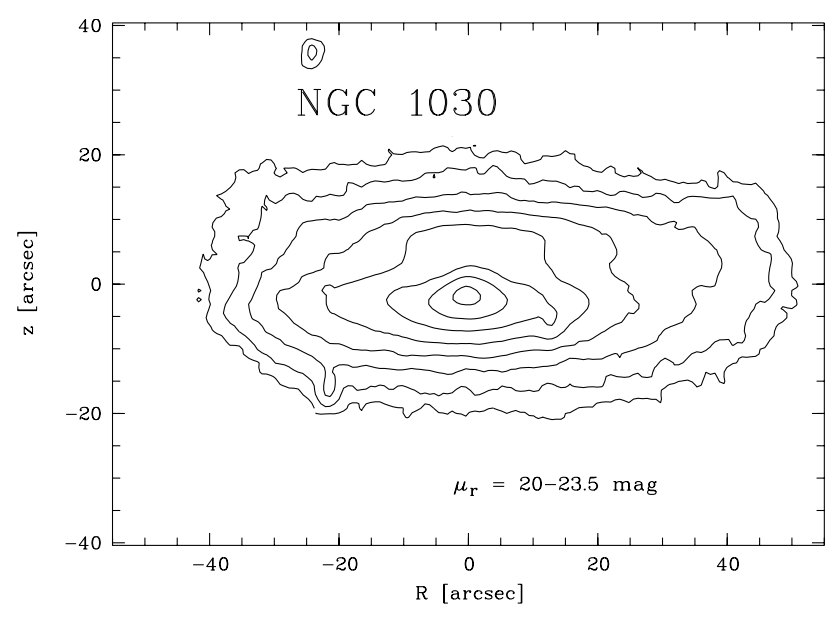

Fig. 4. NGC 1030: Lowell/1.06 m, $15 \mathrm{~min}$ in $r$.

Table 2. Asymmetry of TBBs (RC3-survey).

\begin{tabular}{|c|c|c|c|c|}
\hline & \multicolumn{4}{|c|}{ Galaxies with a } \\
\hline & TBB & TSB & $\begin{array}{c}\text { normal sized } \\
\text { boxy } \\
\text { bulge }\end{array}$ & $\begin{array}{c}\text { normal sized } \\
\text { elliptical } \\
\text { bulge }\end{array}$ \\
\hline asymmetric & 7 & 0 & 14 & 5 \\
\hline symmetric & 6 & 5 & 303 & 394 \\
\hline
\end{tabular}

NGC 1055 (Fig. 5) is already well studied in the literature (e.g. Shaw 1993). The boxy isophotal distortions within the bulge component are spatially extended and form almost a quadratic bulge. While on one side the disk is bending away from the central plane and is clearly separated from the bulge, on the other side bulge and disk overlap. Additionally, the prominent dust lane on the northern side of the galaxy is remarkable.

The bulge of NGC 1589 (Fig. 6) is merged with a thick asymmetric disk. The boxiness is very pronounced in the inner part of the galaxy.

The galaxy ESO 383-005 (Fig. 7) - previously studied by Kemp \& Meaburn (1993) pointing among other features also to the considerable flat bulge - is the latest type (Sbc) in the 


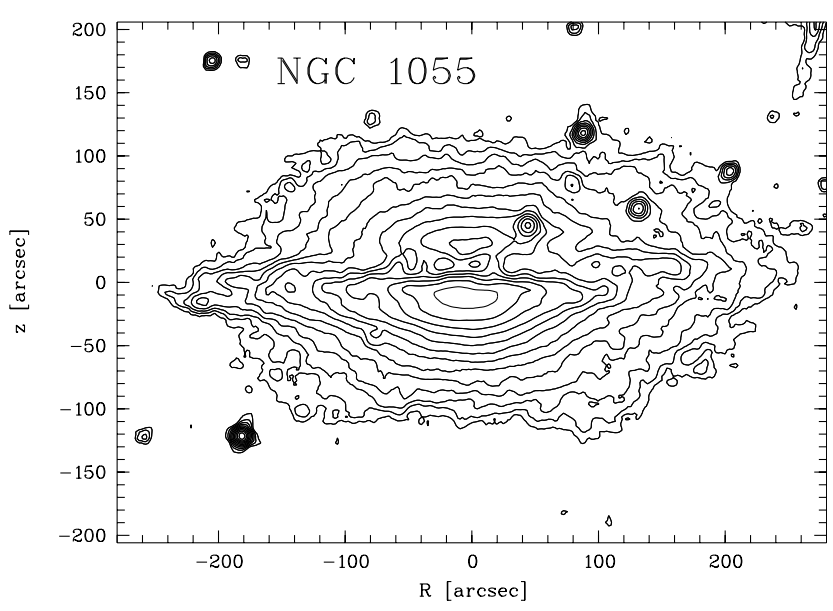

Fig. 5. NGC 1055: contour plot from DSS.

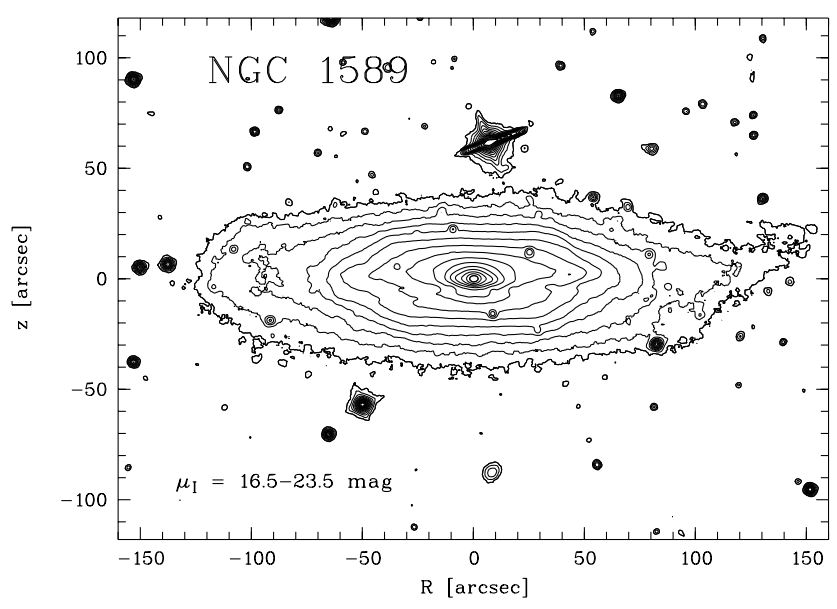

Fig. 6. NGC 1589 has a prominent boxy bulge merging into a thick disk. ESO/1.54 m Danish, 20 min in $I$.

sample of TBBs. In the outer part of this galaxy its warped disk can be well separated from its large boxy bulge. Additionally, the disk is asymmetric in respect to its extent from the center and is tilted with respect to the bulge $\left(\sim 3^{\circ}\right)$ (Dettmar \& Barteldrees 1990a; Lütticke 1996). One unusual substructure in ESO 383-005 is remarkable (Fig. 7) and could be a possible merger remnant.

The boxy bulge of NGC 3573 (Fig. 8) is at low surface brightness levels very asymmetric. On one side of the minor axis the bulge clearly ends and a perturbed warped disk component follows, but on the other side bulge and disk component are merged and largely spread into a diffuse structure which is also asymmetric with respect to the major axis.

The most conspicuous component of ESO 510-013 is its strong warped disk. In the outer regions this twisted disk contains bright clouds of blue stars (Conselice 2001). Additional to a prominent dust lane aligned with the disk, there exists a second less pronounced dust lane which is inclined by $\sim 8^{\circ}$. Their intersection is outside of the galaxy center. As already mentioned in the ESO Press Information (1999) the prominent dust lane and the inner part of the bulge are not well aligned. Especially the position angle of the main axis of the dust lane differ from the main axis of the outer boxy part of the bulge.

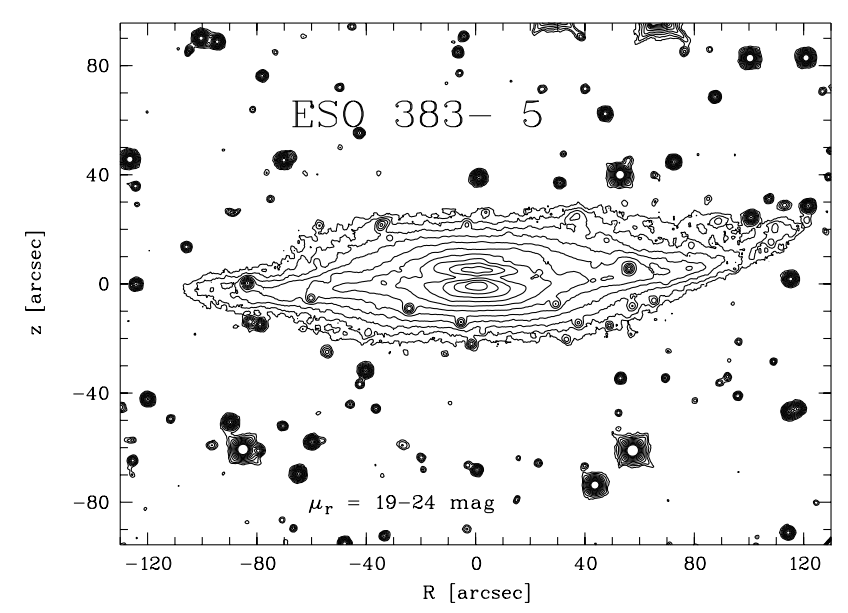

Fig. 7. Latest morphological TBB with type (Sbc): the substructure in ESO 383-005 at $(R, z)=\left(38^{\prime \prime}, 24^{\prime \prime}\right)$ could be possibly a merger remnant. ESO/1.54 $\mathrm{m}$ Danish, $20 \mathrm{~min}$ in $r$.

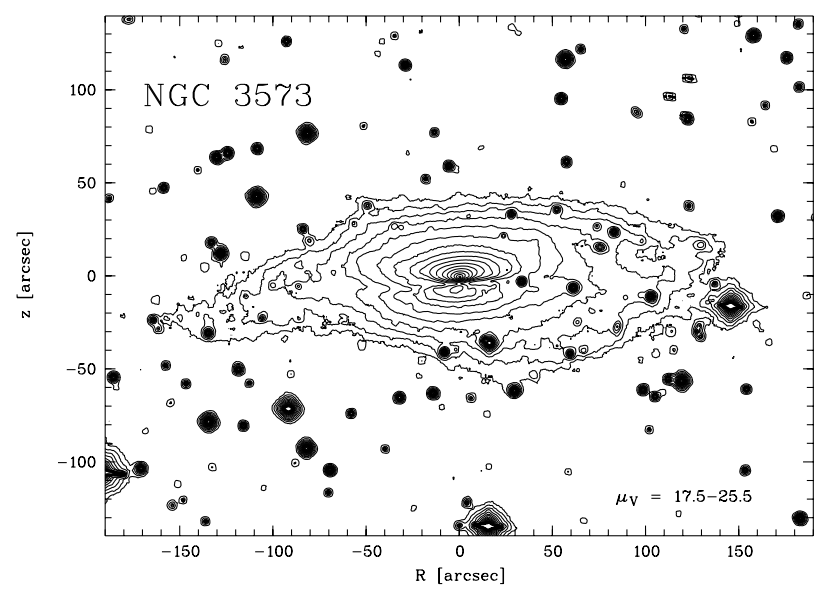

Fig. 8. The asymmetric and strongly disturbed shape of NGC 3573 at low surface brightness outer contours. ESO/1.54 m Danish, $30 \mathrm{~min}$ in $V$.

Since this extensive boxiness is only visible at fainter brightness levels $(\mu>21.6 \mathrm{mag})$, it is possible that Conselice (2001) has not noticed this feature when he is describing ESO 510-13 as galaxy containing "a large spheroidal bulge". On the other side van Driel et al. (2000) also mentioned the "boxy structure" of this galaxy. A further remarkable feature of the bulge of ESO 510-013 is the excess of light on two diagonally opposite edges (possibly marking the accreted satellite track and leading to the classification "System possibly related to polarring galaxies" (PRC D-43, Whitmore et al. 1990).

In NGC 5719 (Fig. 10) the tilted dust lane is remarkable. This dust lane is significantly bent and inclined to the major axis of the galaxy. The boxy structure in the outer parts of the bulge envelops the inner dust distorted bulge.

Peletier \& Balcells (1996) find in NGC 5719 differences in $(B-R)$ and $(U-R)$ between bulge and disk of the order 0.2 mag resp. $0.3 \mathrm{mag}$. These differences are nearly the largest values in their sample of 30 galaxies, although the values are not extraordinary. Balcells \& Peletier (1994) find a typical colour gradient within the bulge reflecting the blueing to the outer parts of the bulge. 


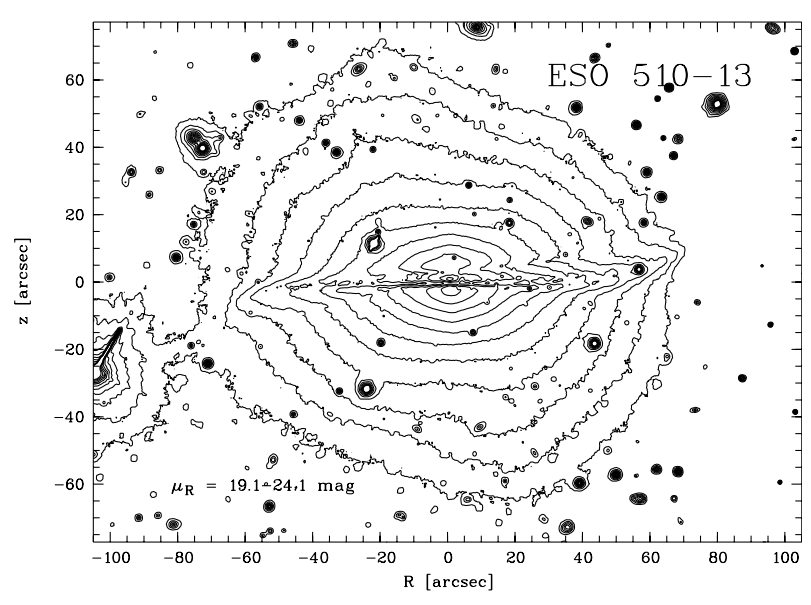

Fig. 9. Contour plot of ESO 510-13 from VLT. Besides the warped dusty disk the enveloping boxy bulge with luminosity excess on the lower-right and upper-left sides is remarkable: $(R, z)=\left(-20^{\prime \prime}, 50^{\prime \prime}\right.$; $\left.20^{\prime \prime},-50^{\prime \prime}\right)$. ESO archive, $5 \mathrm{~min}$ in $R$.

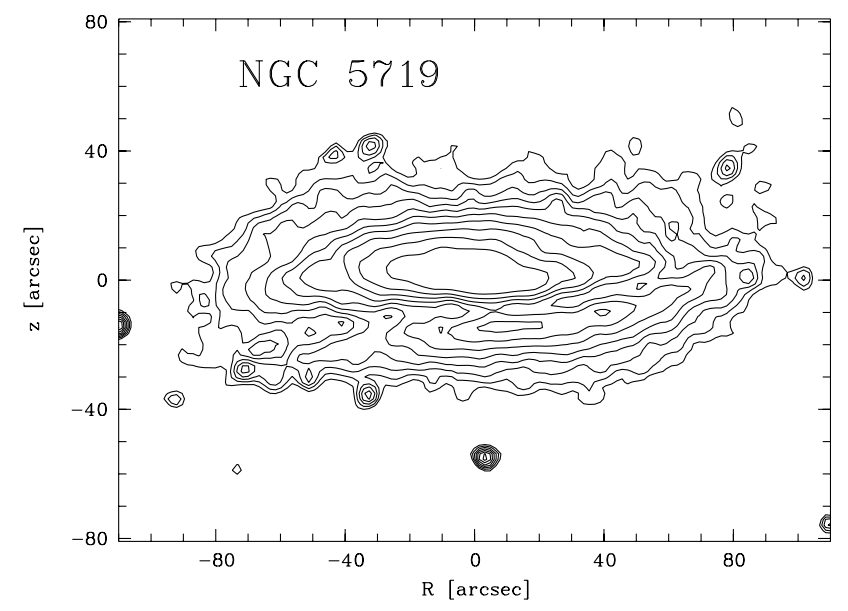

Fig. 10. NGC 5719: contour plot of NGC 5719 from DSS.

UGC 10205 (VV 624) (Fig. 11) is already studied in detail by Reshetnikov \& Evstigneeva (1999) and Vega et al. (1997). Both note that the bright spots in this galaxy (first mentioned by Vorontsov-Velyaminov 1977) belong to an outer equatorial (edge-on) ring. Other interpretations of this structure as a merger remnant (Vorontsov-Velyaminov 1977) or polar ring (Whitmore et al. 1990; van Driel et al. 2000), seem to be very unlikely. Reshetnikov \& Evstigneeva (1999) point also to the asymmetric weak envelope surrounding the galaxy. However, they do not mention its boxy structure at certain contour levels. The asymmetry of the boxy envelope is especially remarkable in the outer parts of the galaxy. Additionally, Reshetnikov \& Evstigneeva (1999) explain an "extended, diffuse structure" at one side of the galaxy, only detectable in deep exposures (not visible in our DSS image, but in Fig. 2 in Reshetnikov \& Evstigneeva 1999), as result of an accretion event.

IC 4745 (Fig. 12) is one of the most prominent member of the galaxies with a TBB and shows a lot of irregularities. Therefore it is surprising that Bell \& Whitmore (1989) do not mention its peculiar structure. The inner bulge system is asymmetric with respect to the disk and the outer part of the bulge

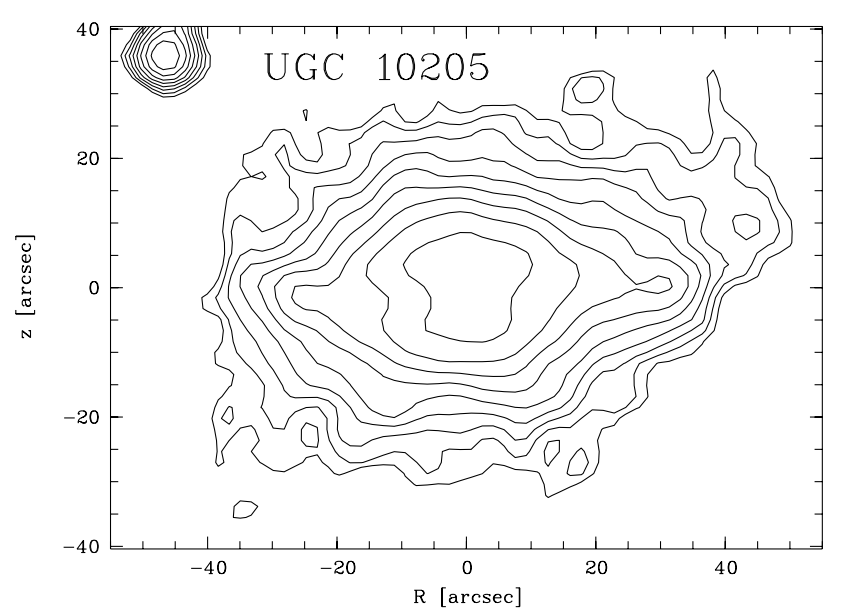

Fig. 11. UGC 10205: contour plot from DSS. The faint extended structure not visible in this image is to the lower left side of the galaxy.

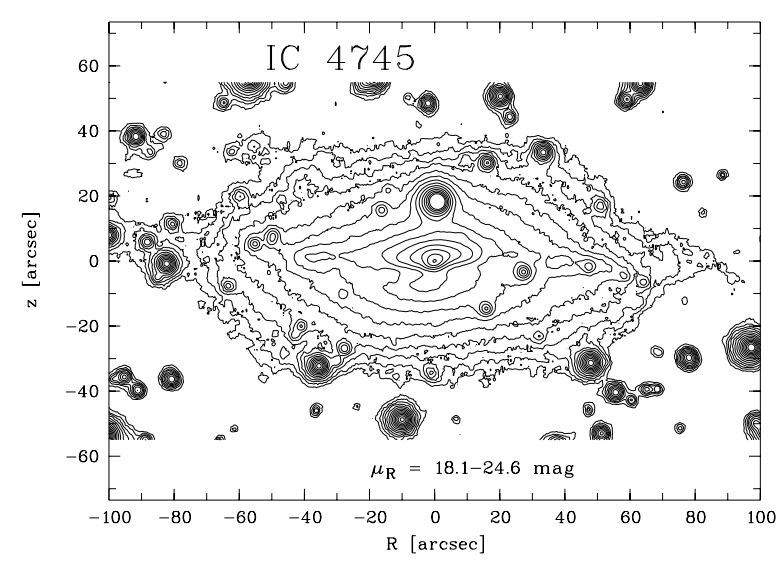

Fig. 12. IC 4745 is the prototype of the galaxies with a TBB because of its many irregularities, substructures, and asymmetries. ESO/1.54 m Danish, 20 min in $R$.

(Dettmar 1989; Dettmar \& Barteldrees 1990a) as shown by a twist of the isophotes visible in NIR images (Lütticke 1999). The boxiness is most pronounced at lower surface brightness levels. Similar to NGC 1055 the disk is on one side more extended and separated from the bulge, while on the other side bulge and disk are merged at larger radial distances. This impression is supported by the fact that the center of the boxy envelope of the bulge is shifted $\left(\sim 5^{\prime \prime}\right)$ to the side of the merged disk/bulge component. Although many bright stars in the foreground obscure the analysis of additional substructures, one feature at $(R, z)=\left(-40^{\prime \prime}, 25^{\prime \prime}\right)$ is notable (Fig. 12). It can be associated with a possible merger remnant. Perhaps related to this structure is a further feature which is in the outer region of the galaxy at $(R, z)=\left(-55^{\prime \prime}, 35^{\prime \prime}\right)$. A dissolving satellite could be an explanation for this structure.

The blueing with radial distance is evident in the $(V-R)$ colour profiles (Fig. 13).

IC 4757 exhibits a boxy bulge extending into a boxy envelope on all contour levels, without showing a normal disk component (Fig. 14).

While its asymmetry in the optical image is difficult to see, it is noticeable in the colour $(B-R)$. The colour difference 


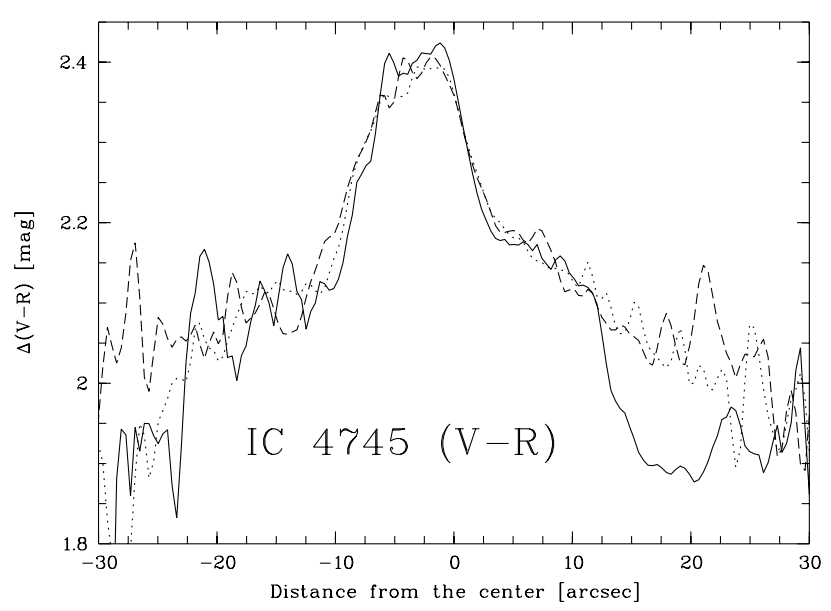

Fig. 13. In cuts of the colour map through the bulge the blue gradient is eminent. Minor axis cut: solid line. Cut with an angle of $60^{\circ}$ to the major axis: dotted line. Cut with an angle of $-60^{\circ}$ : dashed line.

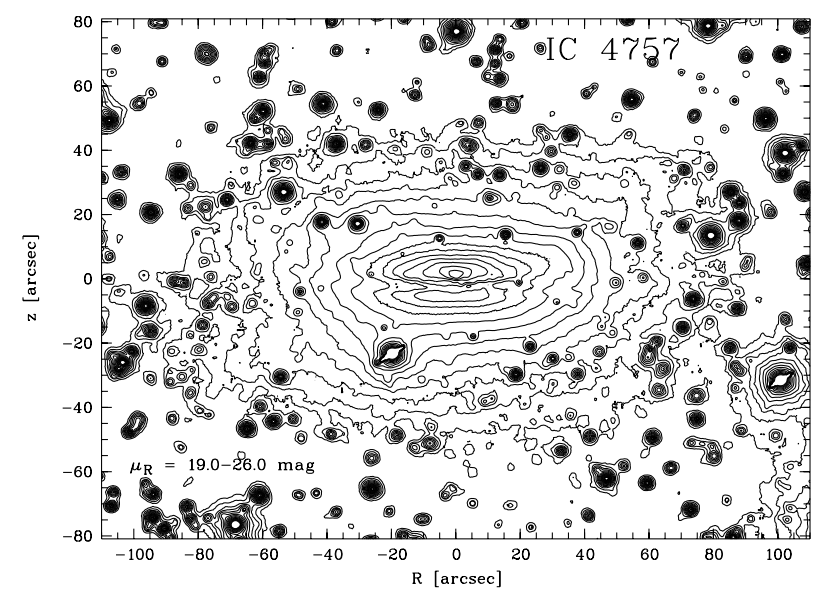

Fig. 14. Deep image of IC 4757: NTT/ESO, $30 \mathrm{~min}$ in $R$.

between the parts of the bulge above and below the dust lane, $\Delta(B-R)=0.2 \mathrm{mag}$, is larger than the colour differences in each part, $\Delta(B-R)=0.1 \mathrm{mag}$ (Fig. 15). This unusual result can not be explained by dust reddening because the red side of the bulge is not affected by the dust lane. Bad alignment between the images of the different filters can also be excluded due to the lack of asymmetric residuals of stars in the colour map (cf. Lütticke 1999).

NGC 7183 (Fig. 16) has a bulge with a disturbed boxy shape and notable asymmetries. In the outer parts the merged disk/bulge component is asymmetrically extended.

\subsection{Luminosity profiles}

Studies in the literature reveal that the luminosity distribution of bulges is well fitted by the Sersic $r^{1 / n}$-law. The shape parameter $n$ is correlated with the Hubble type in the sense that late type galaxies have exponential bulges $(n=1)$ and bulges of early types are closer to the original de Vaucouleurs-law (1948) with $n=4$ (Andredakis et al. 1995; Graham 2001).

The often asymmetric and merged bulge and disk components severely hampers any bulge/disk decomposition.

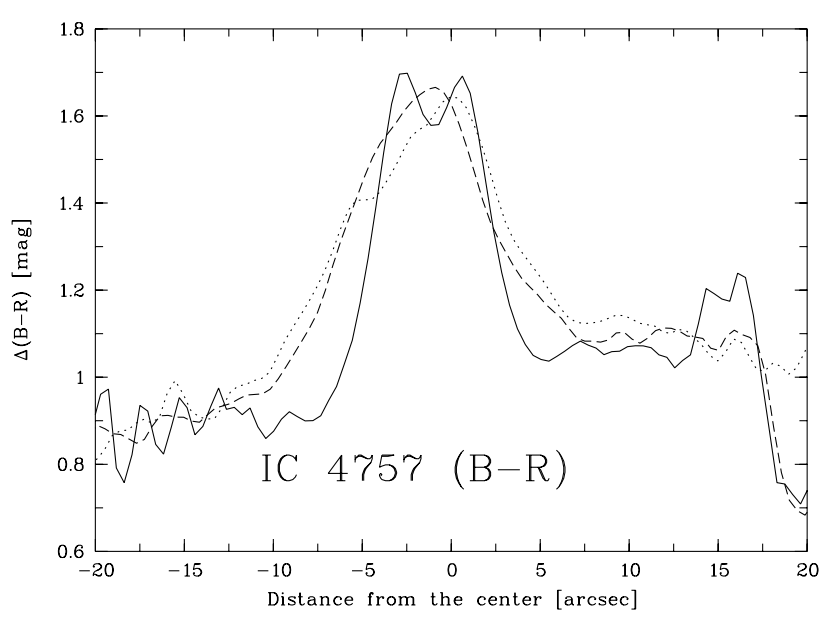

Fig. 15. Color profiles in $(B-R)$ reveal the asymmetry in the bulge. The colour above the plane (positive values of the abscissa) is obviously redder than the colour below the plane. Minor axis cut: solid line. Cut with an angle of $45^{\circ}$ to the major axis: dotted line. Cut with an angle of $-45^{\circ}$ to the major axis: dashed line.

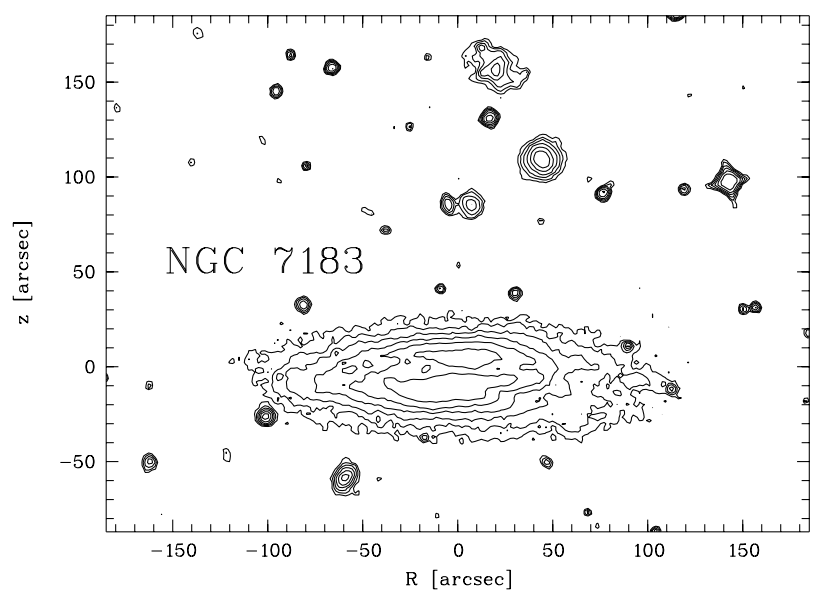

Fig. 16. NGC 7183 and its projected satellite system: $(R, z)=\left(-60^{\prime \prime}\right.$, $\left.-60^{\prime \prime}\right),\left(-5^{\prime \prime}, 85^{\prime \prime}\right),\left(5^{\prime \prime}, 85^{\prime \prime}\right),\left(20^{\prime \prime}, 160^{\prime \prime}\right),\left(110^{\prime \prime}, 45^{\prime \prime}\right)$. Contour plot from DSS.

Therefore it is only possible to determine the shape of the luminosity distribution of the bulge along the minor axis to minimize the influence of the disk in edge-on galaxies. The analysis of the luminosity profiles, testing only $n=1,2,3$ and 4, shows that $n=4$ is only for one (UGC 10205) out of thirteen investigated TBBs the best description. In this galaxy, the minor axis surface brightness plotted against $r^{1 / 4}$ shows two components. Their intersection is at $z= \pm 10^{\prime \prime}$. Three bulges have a shape parameter of $n=3$, while seven bulges, even in early type galaxies (Figs. 17 and 18), follow the $r^{1 / 2}$-law. Two galaxies have even exponential bulges (Table 1). There is no clear correlation for the galaxies with a TBB between $n$ and the Hubble type.

\subsection{Bar detection}

Kinematical studies (Vega et al. 1997; Merrifield \& Kuijken 1999; Garcia-Burillo 2001) and bar detection in radial cuts of NIR images (Lütticke 1999; Vergani et al. 2001b, Paper II) 


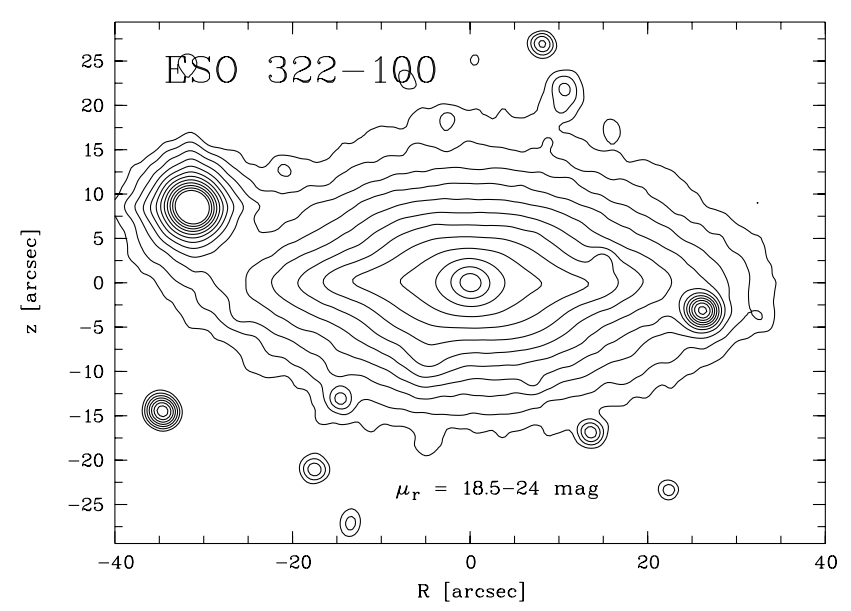

Fig. 17. ESO 322-100: $\mathrm{ESO} / 2.2 \mathrm{~m}, 15 \mathrm{~min}$ in $r$.

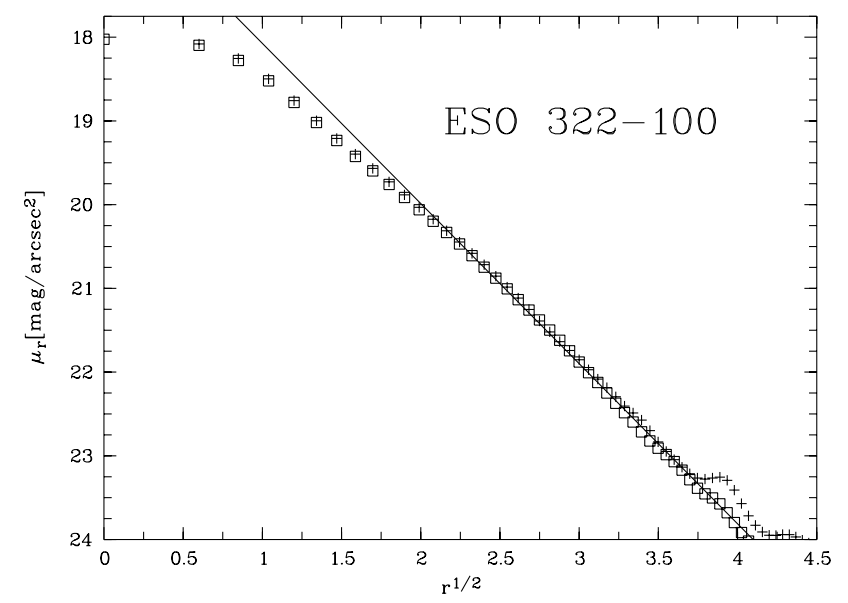

Fig. 18. The TBB of the S0 galaxy ESO $322-100$ is described best by the shape parameter $n=2$. This reveals the luminosity profile along the minor axis in the outer bulge parts. The surface brightness approximately correlates with $r^{1 / 2}$. The cut above the plane is marked by crosses, below the plane by squares.

reveal that the most galaxies with a TBB do not habour a bar. In detail, from nine TBBs studied only one possesses a bar, whereas six do not show a bar signature (Table 3). For two galaxies there are controversial results. Garcia-Burillo (2001) and Vergani et al. (2001b) detect a bar in NGC 1055, Merrifield \& Kuijken (1999) did not. UGC 10205 is denoted as unbarred by Reshetnikov (2003) and Lütticke (1999) while Vega et al. (1997) propose that the galaxy harbours a bar. However, inspecting their data the interpretation of the velocity curve of UGC 10205 by a possible bar seems to be unlikely (cf. Sect. 4.1.2).

\subsection{Environment}

To investigate environmental effects on the galaxies with a TBB, we have examined the large scale as well as the small scale environment.

3033 out of the total sample of 6392 galaxies (47\%) in Garcia (1993) belong to groups, but the fraction of galaxies with a TBB in groups is significantly larger (77\%, Table 1).
Table 3. Bar detection in TBB galaxies.

\begin{tabular}{l|c|ll}
\hline \hline Galaxy & Bar & \multicolumn{2}{|c}{ Reference $^{1}$} \\
& & for a bar & for no bar \\
\hline NGC 1030 & no & & V01 \\
NGC 1055 & $?$ & G01, V01 & M99 \\
NGC 1589 & yes & V01 & \\
UGC 3458 & no & & L99 \\
ESO 506-003 & no & & L99 \\
UGC 9759 & no & & L99, V01 \\
UGC 10205 & $?^{2}$ & V97 & L99, R03 \\
IC 4745 & no & & L99 \\
NGC 7183 & no & & V01 \\
\hline
\end{tabular}

1: G01: Garcia-Burillo (2001), L99: Lütticke (1999), M99: Merrifield \& Kuijken (1999), R03: Reshetnikov (2003), V97: Vega et al. (1997), V01: Vergani et al. (2001b).

2: most likely: no (cf. text).

Table 4. Number of galaxies with projected satellites.

\begin{tabular}{lcc}
\hline \hline & $\begin{array}{c}\text { Galaxies with } \\
\text { a TBB }\end{array}$ & $\begin{array}{c}\text { Galaxies without } \\
\text { a b/p bulge }\end{array}$ \\
\hline no projected satellite & 5 & 21 \\
projected satellite(s) & 16 & 10 \\
\hline
\end{tabular}

A galaxy is here defined as projected satellite, if it is inside a radius of the primary galaxy diameter $\left(1 \times D_{25}\right)$ around this galaxy.

However, a direct comparison with a control sample of non-b/p shaped bulges (drawn from Lütticke 1999) with similar Hubble types ( $\mathrm{S} 0-\mathrm{Sb}$ ) also exhibits a fraction of $67 \%$ of galaxies in groups. This reveals that the rate of galaxies with a TBB in groups is not significantly increased.

In fields around the center of a galaxy with a TBB scanning a radius of $5 \times D_{25}$ resp. $2.5 \times D_{25}$ there is nearly no difference in the number distribution of confirmed companions $\left(\Delta v<1000 \mathrm{~km} \mathrm{~s}^{-1}\right)$ with known velocity (derived from $\mathrm{NED}^{4}$ ) compared to the control sample. E.g., $63 \%$ of the galaxies with a TBB have no companion inside $5 \times D_{25}$ and the mean value of companions is $0.6 \pm 0.2$, while in the control sample the fraction is $68 \%$ with the same mean value.

However, inside $1 \times D_{25}$ around a galaxy with a TBB $84 \%$ of these galaxies have at least one projected satellite visible on DSS images. The control sample only shows a fraction of $32 \%$. This dependence between the existence of nearby satellites and TBB is statistically significant on a $0.1 \%$-level $\left(\chi^{2}\right.$-test $)$ (Table 4).

The nearby satellite system of NGC 7183 with at least five projected galaxies is especially remarkable (Fig. 16). IC 4745 has also a prominent nearby satellite system including at least three galaxies. However, the satellite system has a larger extent than the system of NGC 7183. Furthermore, NGC 3573 has one projected satellite only $2^{\prime}$ and one confirmed companion (ESO 377-017, $\Delta v=22 \mathrm{~km} \mathrm{~s}^{-1}$ ) 12' away.

Determining the normalized interaction index $I$, defined by van den Bergh et al. (1996), the galaxies with a TBB reveal

\footnotetext{
${ }^{4}$ NASA Extragalactic Database.
} 
Table 5. Interaction index.

\begin{tabular}{lccccccc}
\hline \hline sample & $w:$ & 0 & 1 & 2 & 3 & 4 & $I$ \\
\hline $\begin{array}{l}\text { Galaxies with } \\
\text { a TBB }\end{array}$ & 0 & 7 & 10 & 2 & 0 & $1.7 \pm 0.2$ \\
\hline $\begin{array}{l}\text { Galaxies without } \\
\text { alan }\end{array}$ & 26 & 3 & 2 & 0 & 0 & $0.2 \pm 0.1$
\end{tabular}

a b/p bulge

with $w=0$ : objects with no tidal distortion, $w=1$ : objects with possible tidal distortion, $w=2$ : objects with probable tidal effects, $w=3$ : possible merger, $w=4$ : almost certain merger.

a large value of $1.7 \pm 0.2$ (Table 5) which is even larger than the interaction index of the Hubble Deep Field $\left(I_{\mathrm{HDF}}=1.0\right.$, van den Bergh et al. 1996). Regarding the morphological pecularities of the TBBs such a large interaction index for this group of galaxies is not surprising, but it quantifies the characteristic of these galaxies.

\section{Discussion}

\subsection{The origin of the box structure in the TBB}

\subsubsection{Bars?}

Results of different studies (cf. Sect. 3.4) show that more than $60 \%$ of the galaxies with a TBB have no bar. Therefore not all TBBs can be explained by the existence of bars. However, there is the possibility that former bars (which are now dissolved) could be responsible for the boxy structure of the TBBs. It is known that bars can be destroyed by large gas inflow and mass accumulation in the center (Hasan et al. 1993; Friedli \& Benz 1993; Norman et al. 1996; Sellwood \& Moore 1999; Bournaud \& Combes 2002; Berentzen et al. 2003) and boxy bulges arise progressively at the end of the bar life-time (Combes 2003). On the other side, recent studies show that bars are more robust against central mass concentration than previously thought (Shen \& Sellwood 2003).

Is it therefore possible that all TBBs have their origin in dissolved or existing bars?

In general, TBBs are often more box shaped in the outer than in inner part of the bulge in contrast to normal b/p shaped bulges. Regarding simulations the shape of boxy bulges produced by a bar look very different to the observed TBBs in vertical as well as in radial extent (cf. Combes et al. 1990, Paper II; Athanassola \& Misiriotis 2002; Patsis et al. 2002b). Quanfifying this result it is evident that bars can only be responsible for boxy structures smaller than $D_{\text {bar }}$ and for boxy structures with larger vertical extent smaller than $0.7 \times D_{\text {bar }}$ (e.g. Athanassola \& Misiriotis 2002; Patsis et al. 2002b).

Measurements for samples of strong bars give medium relative bar lengths of $D_{\text {bar }} / D_{25}=0.37 \pm 0.03$ (Sab-Sc galaxies, derived from Elmegreen \& Elmegreen 1995) resp. $0.42 \pm 0.05$ (S0-Sb galaxies, derived from Paper II setting $B A L=D_{\text {bar }}$ in galaxies with a peanut-shaped bulge). In the literature there are only a few measurements detecting bar lenghts $\left(D_{\text {bar }} / D_{25}\right)$ larger than 0.5 (Elmegreen \& Elmegreen 1985; Otha et al. 1990; Elmegreen \& Elmegreen 1995; Shaw et al. 1995, Paper II; Erwin \& Sparke 2003). Using a classical method (cf. Erwin \& Sparke 2003) Otha et al. (1990) find the only bar (as far as we know) with a relative length larger than 0.6. Using a new method to quantify the length of a bar, Erwin \& Sparke (2003) find additional four bars with values larger than 0.6.

How about the origin of the TBB for galaxies with a bar detection? In the case of NGC 1055 (cf. Sect. 3.4) the position of the end of the (possible) bar, derived from radial NIR profiles, is in the range of the turn-over point of the rotation curve at a radial distance of $R=30^{\prime \prime}$ (measured at a vertical distance of $z=-15^{\prime \prime}$ below the major axis, well out of the dust lane) (Shaw 1993). Since the box shape of the bulge is most pronounced at $R=120^{\prime \prime}$ (cf. Fig. 5), the existing bar potential cannot explain this box structure. However, another possibility would be to assume that there was a larger (now dissolved) bar present in this galaxy. This could be explained by the evolutionary scenario with bar formation and renewal of Bournaud \& Combes (2002). They show in simulations that former bars are relative larger than later ones. However, the differences of the lenghts of the bars in their simulations are far away from a factor of four, which we would need in the case of NGC 1055 to conclude from an existing bar of a projected length of $R=30^{\prime \prime}$ to a former bar of $R=120^{\prime \prime}$.

In addition, it is not yet clear how shorter bar lenghts after the bar renewal processes fit to an evolution along the Hubble sequence from late- to early-types, since all observations reveal that bars in late-type galaxies are shorter with respect to the galaxy size compared to early-type galaxy bars (Elmegreen et al. 1996; Erwin \& Sparke 2003; Erwin 2003).

To conclude: the small number of observed bars in TBBs, the boxiness of TBBs in their outer parts in comparison to normal b/p bulges, the size of the TBBs in relation to observed bar lenghts, and the lack of any explanation for the large TBB of NGC 1055 by a present or dissolved bar are strong indications against a scenario of TBBs originating by bars.

There is of course the possibility that in some galaxies with a TBB the inner boxy structures could be produced by the bar in the usual way but for the outer boxiness there have to exist another explanation.

Therefore it is not possible that the galaxies with a TBB are only the most early types in the normal $\mathrm{b} / \mathrm{p}$ category which is in general associated with bars. We have found a lot of S0 galaxies with a $\mathrm{b} / \mathrm{p}$ bulge which have small bulges (that means BUL $\left./ D_{25}<0.5\right)$ and do not belong to the class of TBB galaxies (Paper I). In addition, the ratios of the luminosity of the bulges to the total luminosity of galaxies with normal b/p bulges are in the most cases significally larger compared to the TBB galaxies. This is the reason why TBB galaxies are classified in the range $\mathrm{S} 0$ to $\mathrm{Sbc}$, although their bulges have a large spatial size. However, size is not a criterion for classification but luminosity.

\subsubsection{Soft mergers?}

Regarding the merger/accretion scenario for the origin of b/p bulges (cf. Sect. 1) Vergani et al. (2001a) find evidences for a soft merger between NGC 1055 and a satellite analysing 21-cm HI observations. Asymmetries in the distribution and in the kinematics of the neutral hydrogen and particularly the asymmetric clumpy HI emission in the outermost northwestern 
region indicate an unrelaxed structure typical for tidal interactions and accreted material enlarging the bulge. In further three investigated TBBs the $\mathrm{HI}$ is also distributed in a peculiar way. One galaxy has a strong warped disk (ESO 383-005), the other harbours significant non-settled (perhaps counterrotating) gas (IC 4745), and the third exhibits a patchy HI distribution in general (IC 4757) (Vergani et al. 2001c).

The two components of the bulge of UGC 10205 detected in the analysis of the luminosity distribution in UGC 10205 (cf. Sect. 3.3) are in agreement with the multi-component structure of the gas revealed by Vega et al. (1997). They disentangle three kinematically distinct gaseous components for this galaxy. Two of them have quite similar velocity dispersion profiles, but very different velocity curves. Vega et al. (1997) interpret them by a possible bar due to a similarity to velocity curves with a "figure-of-eight" appearance which is the signature of a bar (Kuijken \& Merrifield 1995). They associate the third component to the faint features embedding the galaxy. However, the "figure-of-eight" signature is only very weak and Reshetnikov \& Evstigneeva (1999) suggest a different origin: one gas component is determined by the rotation of the bulge, another by the overall potential of the bulge and disk, and the peculiar inner subsystem is the result of accreted material from a small companion. They support this conclusion by the observation that the star-formation rate is fairly high compared to normal disk galaxies. In addition, they run a numerical simulation of a capture and tidal disruption of a satellite galaxy by UGC 10205 producing the observed asymmetric envelope structure (Reshetnikov \& Evstigneeva 1999).

The evolutionary scenario in which TBBs are formed by soft mergers is also probable for the prototypical case, IC 4745. This galaxy shows many features supporting an accretion scenario: substructures which could be possible merger remnants or merging satellites, a numerous satellite system pointing to a high density of galaxies, a significantly asymmetric envelope, a colour gradient becoming bluer at increasing radial distance, and even asymmetries reflected in the rotation curve on the major axis (Dettmar \& Barteldrees 1990a). All these observations lead to the conclusion that the bulge structure of IC 4745 has been formed just recently and is not yet dynamically settled similar to UGC 10205.

All other galaxies of this class also show at least some indications (cf. Sect. 3.1) for a merger induced origin of the TBBs which is further supported by the generally stronger asymmetries of these galaxies compared to the galaxies without a TBB (Table 2).

The soft merging scenario is able to explain the deformed structures of asymmetric or thick disks and the merged bulge and disk components which are not produced in a bar scenario. The strongly warped dusty disks of the galaxies with a TBB are indications for such recent interactions (Conselice 2001) and the observed tilted disks could also point to the effects of the gravitational forces (however, triaxiality of bulges is also a possible explanation for tilted disks). Furthermore, unusual components and structures in addition to disk and bulge (as e.g. in UGC 10205) and high star formation rates (UGC 10205, Reshetnikov \& Evstigneeva 1999; ESO 510-014, Conselice 2001) are evidences for a recent merger event. Since such events often trigger bars (Noguchi 1987; Gerin et al. 1990; Walker et al. 1996) there is a combination of bars and mergers building TBBs, but the bars do not seem to be responsible for the extended boxy structures (cf. previous section).

The increased fraction of small nearby projected satellites coupled with a large interaction index also supports the conclusion that the environment of galaxies with a TBB has an increased density of small galaxies and former or ongoing accretions of satellites seem to be very likely.

\subsubsection{Alternative scenarios?}

Explaining the box structure only by interactions, as shown by May et al. (1985), external cylindrically symmetric torques are required. However, these torques arise only in very special conditions. Therefore the fraction of such bulges produced in this way is very small or such bulges do not exist at all. Perhaps the boxy bulge of NGC 3573 marked by large distortions and the nearby satellite and companion could be a result of such a special interaction event.

Using $N$-body simulations Patsis et al. (2002a) have shown that boxy edge-on profiles can also be accounted in models of normal non-barred galaxies by the presence of vertical resonances populating stable families of periodic orbits. However, their simulations reveal that the size of the boxy structures are again too small in comparison to the box-size of the TBB galaxies. Therefore this model is not applicable for the TBBs.

\subsection{Merger scenario: Theory and observation}

The origin of the TBBs by accretion events can be theoretically explained by the study of Binney \& Petrou (1985). The special conditions needed for an accretion leading to a boxy bulge in their investigation correspond to the small number of TBBs. Only $3.9 \%$ of 330 galaxies with a b/p bulge or $1.8 \%$ of 734 disk galaxies derived in the RC3 survey have a TBB.

In the accretion scenario by Whitmore \& Bell (1988) a $\mathrm{b} / \mathrm{p}$ bulge arises if the impact of the satellite is at an oblique angle. Dynamical friction begins to shred the companion on an inclined encounter. The gas settles into a new oblique disk and cloud-cloud collisions therein cause rapid star formation. While the individual resulting stars still relatively circularly orbit around the center of the galaxy, the oblique disk spreads out into a precession cone. In projection this cone will appear as an X-shaped structure which superposes the bulge forming the apparent b/p shape. Hernquist \& Quinn (1993) support by their $\mathrm{N}$-body simulations the possibility of the origin of an X-shaped structure by an accretion event. However, the added mass is small in comparison to the disk in their simulation, while Whitmore \& Bell (1988) suggest that for IC 4767 (a galaxy with a normal b/p bulge) the mass in the X-component has the same size as the mass in the disk. Even taken into account that the X-component has its origin not only in accreted material, but also in recently formed stars, the accreted companion must have a large mass in the picture of Whitmore \& Bell (1988). From the theoretical point of view it is likely that any axisymmetry is destroyed (Combes et al. 1990) and boxiness arises 
also in outer parts of a galaxy (Schweizer \& Seizer 1992) after the merging of such a large amount of mass rotating at a high speed (Combes et al. 1990). Therefore an accretion scenario is not likely for galaxies with normal $b / p$ bulges which are very symmetric and harbour the $b / p$ structure in the inner part of the galaxy.

However, the scenario of Whitmore \& Bell (1988) is a possibility for the formation of the TBBs because they show the expected asymmetry after a merger event and the boxiness is often most pronounced at fainter surface brightness levels leading to the fact that accreted low mass satellites can produce the observed boxiness. Evidence for such an evolutionary picture can be found in ESO 383-005, IC 4745, ESO 510-013, and UGC 10205. The first three galaxies have possible merger remnants at one edge of the box respectively a luminosity excess at two opposite edges. IC 4745 shows an additional indication for a former accretion event, namely the predicted X-structure which is visible in $(V-R)$ (Lütticke 1999). UGC 10205 seems to capture material of a disrupted satellite whose remaining structures are still visible.

\subsection{Merger scenario: Consistency check}

As a consistency check for our merger scenario we can compare the observed number of TBB galaxies in our RC3-survey with a roughly calculated number of TBB galaxies expected from a general accretion rate of satellites. At first we have to answer the question which satellites can produce a TBB. We restrict the parameters for our calculation to the two most important ones, namely mass and impact angle. Measuring the angle $\theta$ between the major axis and the ray passing through the edge of the boxy bulge structure (Shaw et al. 1990; Lütticke 1999) and associating this angle with the infall parameter of the satellite orbit we get an estimate for the range of oblique impact angles which are needed to produce a boxy structure (Whitmore \& Bell 1988). We find angles in the range of $\theta=\left[35^{\circ}, 53^{\circ}\right]$, i.e. $\sim 20 \%$ of all angles are suitable if we suppose that all impact angles are equiprobable (Zaritsky \& Gonzalez 1999). To find out which range of satellite masses can produce a TBB we have looked into several simulations in the literature. Walker et al. (1996), Huang \& Carlberg (1997), Velazquez \& White (1999), and Font et al. (2001) analyse minor mergers in which the satellite has $1-4 \%$ of the mass of the parent galaxy (i.e. 10-30\% of the disk mass). The results are disk thickening, warps, small growth of the bulge etc., but no large scale change of the morphology of the bulges. On the other side of the mass scale Barnes (1992) and Bendo \& Barnes (2000) investigate medium-sized mergers with ratio 1:3 between two disk galaxies. The remnant is described as ellipsoidal with fairly regular disk-like kinematics. Since the TBB galaxies are still disk galaxies such mergers are too violent. Reshetnikov \& Evstigneeva (1999) estimate from their observational data that the mass of the merger remnant of the satellite of UGC 10205 is $4 \%$ of the parent galaxy, while the initial mass of the satellite is $\sim 10 \%$ (Reshetnikov 2003). Regarding these studies we suppose that the suitable mass ratio between the mass of satellite and parent galaxy for the generation of a TBB has to be larger than 1:20 and smaller than 1:3. Therefore an appropriate mass fraction of the satellite seems to be $\sim 10-15 \%$ (i.e. 1:10-1:6). Zaritsky \& Rix (1997) propose for such kinds of mergers (satellite has $\sim 10 \%$ of the mass of the parent galaxy) an upper satellite accretion rate of $0.07-0.25$ per Gyr for local spiral galaxies. The large uncertainty in this upper limit is explained in Zaritsky \& Rix (1997). Using this accretion rate as an upper limit for the calculation of the numbers of TBBs in our RC3-survey we get: 734 (galaxies in the survey) $\times 0.07-0.25$ per Gyr $\times 0.2$ (fraction of satellites with suitable impactangle) $=10-37 \mathrm{TBB}$ galaxies per Gyr.

If we further assume that the precession cone building the boxy structure arises in a late phase of a suitable soft merger (cf. Fig. 3 of Hernquist \& Quinn 1989), i.e. in the last quarter of this merger which will take about one Gyr (Balcells 2003), and that the asymmetries as signature for an accretion are visible 1-2 Gyr (Zaritsky \& Rix 1997; Reshetnikov \& Evstigneeva 1999; Balcells 2003) we estimate that the asymmetric TBB galaxies have a lifetime of $\sim 2$ Gyr. Therefore we get in summary an upper limit for our RC3-survey of 20-74 asymmetric TBB galaxies.

Compared to the seven detected asymmetric TBB galaxies our observations are below this upper limit. A calculation of the upper limit for the number of all (asymmetric and symmetric) TBB galaxies is not possible since we do not yet know how long their thick boxy structure exists, i.e. if there are other mechanisms, such as subsequent mergers or interactions, which can destroy or smooth a TBB.

\subsection{TBB as a stage of secular evolution}

The luminosity distributions of the TBBs are marked by the lack of any correlation between the shape parameter $n$ and the Hubble type and by the often best description of the bulges by a $r^{1 / 2}$-law, also in early type galaxies. An interpretation for the non-existing correlation is that the TBBs could be involved into recent formation processes in which the Hubble type is changing. This scenario is supported by studies revealing that an accretion of a satellite leads to a growing bulge and to an increasing profile index $n$ proportional to the satellite mass (Andredakis et al. 1995; Aguerri et al. 2001). Additionally, such an interpretation is in accordance with the unusual prominent dust lanes in 18 of the 19 TBB galaxies (even in S0-galaxies), since normally such dust lanes are typical for later types. Therefore we conclude that TBB galaxies are in-between two positions in the Hubble sequence. We suppose that galaxies with a TBB could be in a process in which the Hubble type is changing $(\Delta T=2-4)$. In this way theories of secular evolution including transformation of later Hubble types into earlier (e.g. Pfenniger 2000) get observational support.

Regarding the asymmetric TBBs and the TSBs it is possible that the boxiness depends on the aspect angle. Another, additional explanation for the degree of boxiness is given by Schweizer \& Seizer (1992). Their results reveal that boxiness could be related to the time of the last merger event. This is supported by the fact that the boxiness of the rather 
symmetric TBBs is less pronounced in the outer bulge part than in the asymmetric TBBs. In such a scenario the TBBs will become dynamically settled, the bulges develop into spheroidally shaped and symmetries, and relicts of the merger are then only features at very low surface brightness. This picture gets further support by the detection of such extended features at $\sim 28 B$-mag $\operatorname{arcsec}^{-2}$ in "normal" early type disk or TSB galaxies (e.g. Sombrero Galaxy) (Malin \& Hadley 1999).

\subsection{Summary}

In a merger sequence arranged by the effects of a merger on the central component of the parent disk galaxy the mergers resulting in TBBs can be integrated as follows: the smallest mergers have almost no effect (possibly small growth of the bulge) or let arise bars triggering box- or peanut shaped bulges, stronger mergers with an oblique impact angle result in TBBs, large mergers in elliptical galaxies, and the heaviest collisions destroy galaxies. Thus galaxies with a TBB stand between "normal" disk or lenticular galaxies and elliptical galaxies with small disks in respect of the mass of the accreted companion. Regarding the small fraction of TBBs we suppose that mergers resulting in TBBs are only a secondary process for the secular evolution of disk galaxies.

\section{Conclusion}

We present and characterise a new class of disk galaxies. They are defined by:

- Unusually large bulges: BUL/ $D_{25}>0.5$.

- Box-shaped bulges: bulge type 3 or 2 (Paper I).

We call the bulges of these galaxies "Thick Boxy Bulges" (TBBs) according to their appearance. Using DSS, CCD, and NIR data we derive the following results:

- $2 \%$ of all disk galaxies (S0-Sd) belong to the class of TBB galaxies.

- $4 \%$ of all galaxies with a $\mathrm{b} / \mathrm{p}$ bulge have a TBB.

- The extent of the box shape in TBBs seems to be too large to result from a bar potential.

- In general the morphology of the TBB galaxies is disturbed and a large fraction of these galaxies is very asymmetric.

- There exist two kinds of b/p bulges. The "normal" b/p bulges (96\%) are triggered by bars, while the rest - the TBBs - have most likely their origin in accretion of satellites (soft mergers). Bars can also exist in TBB galaxies, or have been present and are now dissolved, however, they seem to be only responsible for the inner and not for the extended boxy structures of TBBs.

The merging scenario is especially supported by many morphological peculiarities (e.g. merger remnants) of the TBB galaxies. Additionally, we propose a possible ongoing change of the Hubble type in galaxies with a TBB. Our studies of colours, environment, luminosity and dust distribution and recent studies of kinematics and HI (Vergani et al. 2001a and c) give further indications for a merger scenario. More evidences for such a scenario come from the comparison between theories of soft merging and our observations, the detailed analysis of the TBB galaxy UGC 10205 (Reshetnikov \& Evstigneeva 1999), and the detection of high star formation rates in two TBB galaxies. The number of observed TBB galaxies is consistent with an upper limit derived from general satellite accretion rates. However, further investigations about star formation history and simulations of minor mergers are needed for a complete understanding of the formation process and the lifetime of TBBs.

Acknowledgements. Part of this work was supported by the Deutsche Forschungsgemeinschaft, DFG. This work is partly based on observations obtained at ESO/La Silla, DSAZ/Calar Alto, and Lowell Observatory. Furthermore, this research has made use of the VLT Science Archive (used observations were made with ESO Telescopes at the Paranal Observatory under programme ID 60. A-9203 (A).) and the NASA/IPAC Extragalactic Database (NED) which is operated by the Jet Propulsion Laboratory, California Institute of Technology, under contract with the National Aeronautics and Space Administration. This research also uses the Digitized Sky Survey (DSS) based on photographic data obtained using Oschin Schmidt Telescope on Palomar Mountain and The UK Schmidt Telescope and produced at the Space Telescope Science Institute. We thank M. Balcells for the stimulating discussion about the merger scenario for TBB galaxies, V. Reshetnikov for his comments on UGC 10205, and F. Combes for suggestions in her referee report.

\section{References}

Aguerri, J. A. L., Balcells, M., \& Peletier, R. F. 2001, A\&A, 373, 786 Andredakis, Y. C., Peletier, R., \& Balcells M. 1995, MNRAS, 275, 874

Athanassoula, E., \& Misiriotis, A. 2002, MNRAS, 330, 35

Balcells, M. 2003, priv. comm.

Balcells, M., \& Peletier, R. 1994, AJ, 107, 135

Barnes, J. E. 1992, ApJ, 331, 699

Barnes, J. E. 1999, Galaxy Interactions, in Galaxy Dynamics, ed. D. R. Merritt, M. Valluri, \& J. A. Sellwood, ASP Conf. Ser., 182, 463

Barteldrees, A., \& Dettmar, R.-J. 1994, A\&AS, 103, 475

Bell, M., \& Whitmore, B. C. 1989, ApJS, 70, 139

Bendo, G. J., \& Barnes, J. E. 2000, MNRAS, 316, 315

Berentzen, I., Athanassoula, E., Heller, C. H., \& Fricke, K. J. 2003 MNRAS, 341, 343

Binney, J., \& Petrou, M. 1985, MNRAS, 214, 449

Bournaud, F., \& Combes, F. 2002 A\&A, 392, 83

Bureau, M. 1998, Bars in Edge-On Spiral Galaxies, Ph.D. Thesis, The Australian National University

Bureau, M., \& Freeman, K. C. 1999, AJ, 118, 126

Combes, F. 2000, Bulge Formation, in Building Galaxies: from the Primordial Universe to the Present, Proceedings of Rencontres de Moriond, ed. F. Hammer, T. X. Thuan, V. Cayatte, B. Guiderdoni, \& J. Tran Thanh Van, 413

Combes, F. 2003, priv. comm.

Combes, F., Debbasch, F., Friedli, D., \& Pfenniger, D. 1990, A\&A, 233, 82

Conselice, C. 2001, http://heritage.stsci.edu/2001/23/ supplemental.html

Dettmar, R.-J. 1989, Box- and Peanut-Shaped Bulges of Disk Galaxies, in The World of Galaxies, ed. H. G. Corwin, \& L. Bottinelli (Springer), 229 
Dettmar, R.-J., \& Barteldrees, A. 1990a, Substructure and asymmetries in bulges, in ESO/CTIO Workshop on Bulges of Galaxies, ed. B. J. Jarvis, \& D. M. Terndrup (ESO), 255

Dettmar, R.-J., \& Lütticke, R. 1999, Do some bulges result from merging?, The Third Stromlo Symposium: The Galactic Halo, ed. B. K. Gibson, T. S. Axelrod, \& M. E. Putman, ASP Conf. Ser., 165, 95

de Vaucouleurs, G. 1948, Ann. Astrophys., 11, 247

de Vaucouleurs, G., de Vaucouleurs, A., Corwin, Jr., H. G., Buta, R. J., \& Fouqué, P. 1991, Third Reference Catalogue of Bright Galaxies (RC3) (Springer)

Elmegreen, B. G., Elmegreen, D. M., Chromey, F. R., Hasselbacher, D. A., \& Bissell, B. A. 1996, AJ, 111, 2233

Elmegreen, B. G., \& Elmegreen, D. M. 1985, ApJ, 288, 438

Elmegreen, D. M., \& Elmegreen, B. G. 1995, ApJ, 445, 591

Erwin, P. 2003, priv. comm

Erwin, P., \& Sparke L. S. 2003, ApJS, 146, 299

ESO Press Information 1999, http://www.eso.org/outreach/ press-rel/pr-1999/phot-20-99.html

Fisher, D., Illingworth, G., \& Franx, M. 1994, AJ, 107, 160

Font, A. S., Navarro, J. F., Stadel, J., \& Quinn T. 2001, ApJ 563, 1

Friedli, D., \& Benz, W. 1993, A\&A, 268, 65

Garcia, A. M. 1993, A\&AS, 100, 47

Garcia-Burillo, S. 2001, priv. comm.

Gerin, M., Combes, F., \& Athanassoula, E. 1990, A\&A, 230, 37

Graham, A. W. 2001, AJ, 121, 820

Hasan, H., Pfenniger, D., \& Norman, C. 1993, ApJ, 409, 91

Hernquist, L. 1993, ApJ, 409, 548

Hernquist, L., \& Quinn, P. J. 1989, ApJ, 342, 1

Huang, S., \& Carlberg, R. G. 1997, ApJ, 480, 503

Kemp, S. N., \& Meaburn, J. 1993, A\&A, 274, 19

Kuijken, K., \& Merrifield, M. R. 1995, ApJ, 433, L13

Lütticke, R. 1996, Flächenphotometrische Untersuchungen zu Eigenschaften von "box/peanut"-bulges in "edge-on" Scheibengalaxien, Diploma Thesis, Ruhr-Universität Bochum

Lütticke, R. 1999, Box and Peanut Shaped Bulges, Ph.D. Thesis, Ruhr-Universität Bochum

Lütticke, R., \& Dettmar, R.-J. 1999, A New Class of Bulges, in The Formation of Galactic Bulges, ed. C. M. Carollo, H. C. Ferguson, \& R. F. G. Wyse (Cambridge University Press), 119

Lütticke, R., Dettmar, R.-J., \& Pohlen, M. 2000a, A\&AS, 145, 405 (Paper I)

Lütticke, R., Dettmar, R.-J., \& Pohlen, M. 2000b, A\&A, 362, 435 (Paper II)

May, A., van Albada, T. S., \& Norman, C. A. 1985, MNRAS, 214, 131

Marlin, D., \& Hadley, B. 1999, Observational Evidence of Interactions in Bright, Nearby Galaxies, ed. D. R. Merritt, M. Valluri, \& J. A. Sellwood, ASP Conf. Ser., 182, 445

Merrifield, M., \& Kuijken, K. 1999, A\&A, 345, L47

Mihos, J. C., Walker, I. R., Hernquist, L., de Oliveira, C. M., \& Bolte, M. 1995, ApJ, 447, L87

Noguchi, M. 1987, MNRAS, 228, 635
Norman, C. A., Sellwood, J. A., \& Hasan, H. 1996, ApJ, 462, 114

Otha, K., Hamabe, M., \& Wakamatsu, K.-I. 1990, ApJ, 357, 71

Patsis, P. A., Athanassoula, E., Grosbol, P., \& Skokos, C. 2002a, MNRAS, 335, 1049

Patsis, P. A., Skokos, C., \& Athanassoula, E. 2002b, MNRAS, 337, 578

Peletier, R., \& Balcells, M. 1996, AJ, 111, 2238

Pfenniger, D. 1999, Ap\&SS, 269, 149

Pfenniger, D. 2000, Evolution Time-scales in the Hubble Sequence, ed. F. Combes, G. A. Mamon, \& V. Charmandaris, ASP Conf. Ser., 197, 413

Pohlen, M. 2001, Structures of Galactic Stellar Disks, Ph.D. Thesis, Ruhr-Universität Bochum

Pohlen, M., Dettmar, R.-J., Lütticke, R., \& Schwarzkopf, U. 2000, A\&AS, 144, 405

Raha, N., Sellwood, J. A., James, R. A., \& Kahn, F. D. 1991, Nature, 352,411

Reshetnikov, V. P. 2003, priv. comm.

Reshetnikov, V. P., \& Evstigneeva, E. A. 1999, Astron. Rep., 43, 367

Rowley, G. 1988, ApJ, 331, 124

Schweizer, F., \& Seizer, P. 1992, AJ, 104, 1039

Sellwood, J. A., \& Moore, E. M. 1999, ApJ, 510, 125

Shaw, M. A. 1993, A\&A, 280, 33

Shaw, M. A., Axon, D. J., Probst, R., \& Gatley, I. 1995, MNRAS, 274, 369

Shaw, M. A., Dettmar, R.-J., \& Barteldrees, A. 1990, A\&A, 240, 36

Shen, J., \& Sellwood, J. A. 2003, ApJ, in press [astro-ph/0310194]

Skiff, B. 1999, priv. comm.

van den Bergh, S., Abraham, R., Ellis, R. S., et al. 1996, AJ, 112, 359

van Driel, W., Arnaboldi, M., Combes, F., \& Sparke, L. S. 2000, A\&AS, 141, 385

Vega, J. S., Corsini, E. M., Pizzella, A., \& Bertola, F. 1997, A\&A, 324, 485

Velazquez, H., \& White, S. D. M. 1999, MNRAS, 304, 254

Vergani, D., Dettmar, R.-J., \& Klein, U. 2001a, The HI kinematics of a Thick Boxy Bulge Galaxy: NGC 1055, in Dwarf Galaxies and their Environment, ed. K.S. de Boer, R.-J. Dettmar, \& U. Klein (Shaker Verlag), 255

Vergani, D., Dettmar, R.-J., \& Klein, U. 2001c, Multi-Wavelength Studies of Merging Bulge Galaxies, Astronomische Gesellschaft Abstract Series, 18, 553

Vergani, D., Pohlen, M., Lütticke, R., \& Dettmar, R.-J. 2001b, Thick Boxy Bulges in NIR, in Dwarf Galaxies and their Environment, ed. K.S. de Boer, R.-J. Dettmar, \& U. Klein (Shaker Verlag), 251

Vorontsov-Velyaminov, B. A. 1977, A\&AS, 28, 1

Walker, I. R., Mihos, J. C., \& Hernquist, L. 1996, ApJ, 460, 121

Whitmore, B. C., \& Bell, M. 1988, ApJ, 324, 741

Whitmore, B. C., Lucas, R. A., McElroy, D. B., et al. 1990, AJ, 100, 1489

Zaritsky, D., \& Gonzalez, A. H. 1999, PASP, 111, 1508

Zaritsky, D., \& Rix, H.-W. 1997, ApJ, 477, 118 